Part of Journal of Research of the National Bureau of Standards, Volume 28, June 1942

\title{
DETERMINATION OF CARBON AND HYDROGEN IN BONE BLACK AND OTHER CHARS ${ }^{1}$
}

\author{
By Victor R. Deitz and Leland F. Gleysteen ${ }^{2}$
}

\section{ABSTRACT}

The carbon and hydrogen contents of samples of bone chars, charcoals, and vegetable carbons are determined by combustion in oxygen, the resultant carbon dioxide and water being weighed. The procedure for the handling of such highly adsorptive substances is set forth with a description of the necessary apparatus.

A simple procedure is adopted to bring each sample for analysis to a constant weight. This consists in exposure of the sample to air saturated with water in an exsiccator for 18 hours, and subsequent heating in a helium atmosphere at $105^{\circ} \mathrm{C}$ for 18 hours. In the determination of the carbon, corrections are made for the carbonate remaining in the ash from the combustion and for the carbonate and the adsorbed carbon dioxide contained in the original sample. The results are tabulated to give these separate contributions to the total carbon. A comparison is made with results for the same materials obtained by the determination of the loss upon ignition of the acid-washed residue of each sample.

\section{CONTENTS}

Page

I. Introduction

II. Preparation of the sample for analysis

1. Sampling

2. Handling of the sample

III. Method A: Combustion in oxygen. 798

1. Apparatus _....... 798

2. Procedure

3. Determination of carbon dioxide in the ash from combustion and in the original substance........ 800

IV. Method B: Loss upon ignition.

V. Discussion of results.

VI. References

\section{INTRODUCTION}

The work described in this paper is part of a research project undertaken at the National Bureau of Standards by the United States Cane Sugar Refiners and the Bone Char Manufacturers to study the physical and chemical properties of bone char and a variety of other carbon-containing adsorbents used in the sugar industry. The ability of such materials to adsorb large quantities of gases and to remove colored substances from solution indicates that carbon itself may be the principal seat of the adsorptive capacity. Among the carboncontaining substances available to industry the carbon content varies from approximately 5 percent in some bone blacks to 90 percent in some vegetable and other activated carbons. It is believed that

\footnotetext{
${ }^{1}$ A part of the material in this paper was presented before the Division of Sugar Chemistry and Technology of the American Chemical Society at the Atlantic City meeting in September 1941.

2 Research Associates at the National Bureau of Standards, representing the United States Cane Sugar Refiners and Bone Char Manufacturers.
}

$$
455653-42-9
$$


two general factors determine the extent of adsorption or "activity," namely (1) the quantity of carbon present, and (2) the specific surface and the physical state of the carbon surface. It is, therefore, important to have accurate data on the percentage of carbon of the chars which have been assembled for extensive study.

Two methods are available for the determination of carbon: (1) The sample may be burned in an atmosphere of oxygen and the resultant carbon dioxide weighed; (2) the carbonaceous residue remaining after extraction with hydrochloric acid may be weighed and then ignited in a tared crucible. The loss in weight upon ignition has been considered to be equivalent to the weight of carbon in the sample. The former method is more fundamental and is required for accurate work in view of the known presence of sulfur, nitrogen, hydrogen, and oxygen compounds with carbon in the original sample.

A search of the literature has failed to disclose any report of the determination of the carbon content of bone chars by combustion of the sample in oxygen and the determination of the resultant carbon dioxide. This method has, however, been used for the analysis of vegetable charcoals by Barker [1] ${ }^{3}$ and for the analysis of coal and coke by the Bureau of Mines [2]. Many of the chars, especially bone chars, contain quantities of ash, which may introduce errors by combining with carbon dioxide to form carbonates. Moreover, there are both adsorbed carbon dioxide and carbonates in the original sample which must be determined. It is therefore, necessary to perform auxiliary analyses to correct for these effects.

This paper presents representative data obtained by both the "Combustion in Oxygen" and the "Loss upon Ignition" methods, the latter being the one in common use in sugar refinery laboratories. The results for 19 samples are included, namely 8 bone chars, 2 charcoals, 7 vegetable carbons, a sample of spectroscopic graphite, and a sample of petroleum coke.

\section{PREPARATION OF THE SAMPLE FOR ANALYSIS}

\section{SAMPLING}

The samples for analysis are from materials which can hardly be considered to be of uniform composition. In addition to the heterogeneity of the original natural sources, there are heterogeneities induced by possible nonuniform treatment in their manufacture. Where necessary, all the samples received from the manufacturer or the refinery were ground in a glazed-porcelain ball mill. One-inch pebbles of quartz were used to grind the softer vegetable carbons, steel balls to grind the bone chars. The portions held by a 35-mesh screen were reground and the "fines" passed by an 80-mesh screen were discarded, thus eliminating dust and possible foreign material. Each of these samples was thoroughly mixed on a large cloth by "rolling" the material from one corner to another several hundred times. They were then stored in narrow-necked, 2-gallon bottles and securely stoppered. These samples were used for the present experiments and are to be used for future investigations. All the results apply to these stored samples, thus eliminating any complications which might be attributed to a segregation of the material due to selective crushing

\footnotetext{
${ }^{3}$ Numbers in brackets indicate the literature references at the end of this paper.
} 
of some of the constituents finer than others which could have occurred in the grinding and sieving operations [11]. Granular material is preferred for the stored samples, because finer material would be undesirable in certain projected experiments, and, moreover, because granular material can always be reduced to any particle size desired.

For the present investigation, samples were desired which were more finely ground than the 35- to 80-mesh materials. Therefore, $100 \mathrm{~g}$ of each of the stored samples was ground in an agate mortar to pass an 80-mesh screen. All of this material was retained, thus reducing the danger of nonrepresentative sampling. A sample of about $0.2 \mathrm{~g}$ of the finely ground material was taken for each analysis.

\section{HANDLING OF THE SAMPLE}

It is obviously necessary that the weight of the samples taken for analysis shall be referred to some state which is reproducible. This

TABLE 1.-Comparison of drying methods

\begin{tabular}{|c|c|}
\hline \multicolumn{2}{|l|}{ I. Drying in air at $150^{\circ} \mathrm{C}$} \\
\hline $\begin{array}{l}\text { Initial weight of sample. } \\
\text { Total loss in weight after: } \\
1 \text { day } \\
2 \text { days } \\
3 \text { days } \\
5 \text { days } \\
7 \text { days }\end{array}$ & $\begin{array}{r}\text { Grams } \\
3.1107 \\
0.1007 \\
.1067 \\
.1096 \\
.1118 \\
.1153\end{array}$ \\
\hline \multicolumn{2}{|l|}{ II. Char moistened and dried in helium at $105^{\circ} \mathrm{C}$} \\
\hline $\begin{array}{l}\text { Initial weight of sample } \\
\text { Total loss in weight after: } \\
1 \text { day days } \\
3 \text { days } \\
4 \text { days }\end{array}$ & $\begin{array}{r}0.22634 \\
.00462 \\
.00458 \\
.00464 \\
.00464\end{array}$ \\
\hline
\end{tabular}

requirement is difficult to meet with a highly adsorbent material like charcoal. It is not possible to bring these samples to constant weight by heating them in an air oven at temperatures from $105^{\circ}$ to $150^{\circ} \mathrm{C}$ for periods from a day to a week, as may be seen from the data in part I of table 1. Stanton, Fieldner, and Selvig [2] state that 1 hour in a current of dry air at $105^{\circ} \mathrm{C}$ suffices in the analysis of coal and coke. Barker [1] dried samples of charcoal in dry nitrogen at $150^{\circ} \mathrm{C}$ for 2 hours and then in a vacuum at $300^{\circ} \mathrm{C}$ for 2 hours, the vacuum being obtained with a rotary oil pump. The dry sample was then removed and placed in a stoppered bottle, weighed, and again transferred to the combustion tube. In the evacuation step the danger of losing the finer dust particles is a serious complication for powdered materials.

It has been found possible in this work to obtain reproducible conditions by the following procedure: The char sample, contained in a platinum boat, is first placed in a closed container in contact with an atmosphere saturated with water vapor for about 18 hours. When subsequently dried in a stream of purified helium at $105^{\circ} \mathrm{C}$ (fig. 1), a constant weight is attained in a single day. This behavior is illustrated by the data in part II of table 1, which is typical for the substances investigated. 


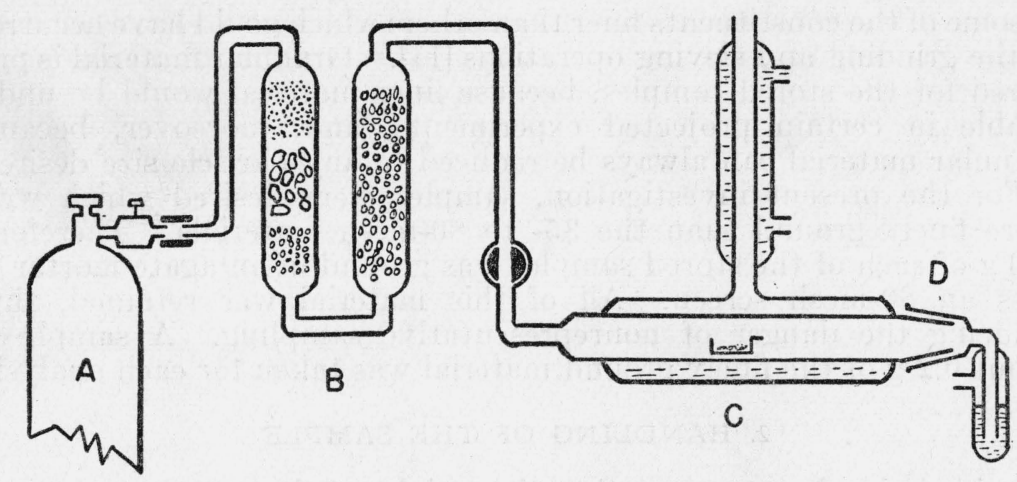

Figure 1.-Apparatus for drying chars to a constant and reproducible weight.

$A$, helium tank; $B$, purifying adsorbents; $C$, reflux drying oven; $D$, ground joint to fit weighing tube.

A plausible explanation of the behavior may be found in the characteristics of the water-adsorption isotherm on charcoals. It has been shown by many investigators, for example by Coolidge [3], that the adsorption isotherms of water are strongly convex to the pressure axis. Therefore, while the first exposure to saturated water vapor results in the displacement of foreign gases from the surface, the subsequent exposure to the stream of dry helium at $105^{\circ} \mathrm{C}$ removes this water, and from the nature of the adsorption isotherm this removal is, for practical purposes, reproducible. It may be pointed out that no significant variation was found in the data for hydrogen content.

Several experiments were performed in which the sample was saturated with ethyl alcohol vapor and subsequently placed in a stream of dry helium at $105^{\circ} \mathrm{C}$. The alcohol is not completely desorbed, as shown by the increased weight of the sample over that obtained by the water-vapor procedure, and as shown by the higher carbon content found upon combustion of such treated samples. This behavior may be expected in view of the fact that the adsorption isotherms of alcohol on chars are strongly concave to the pressure axis and, therefore, appreciable amounts may be retained at relatively low partial pressures of the ethyl alcohol.

\section{METHOD A: COMBUSTION IN OXYGEN}

Samples which have been dried to constant weight with such difficulty must evidently be weighed and handled under conditions which will prevent the readsorption of water vapor and carbon dioxide and of other adsorbable vapors which may be in the air of the laboratory. This has been realized with the use of a special weighing bottle illustrated in figure 2. Since the success of the determination depends on certain details, the procedure is outlined below.

\section{APPARATUS}

The apparatus used to burn the samples was patterned after that described by Wing [4] and by. Washburn, Bruun, and Hicks [5] and is illustrated diagrammatically in figure 3 . The oxygen was purified by passing it first through a preheater packed with copper oxide main- 
tained at a temperature higher than that of the combustion tube proper, and then through absorption tubes containing Ascarite to remove carbon dioxide and phosphorus pentoxide to remove water vapor.

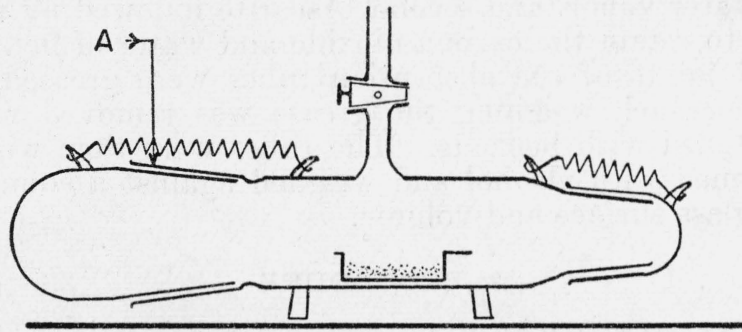

FIgURE 2.-Special weighing tube for handling adsorbent materials.

$A$, ground joint to fit both combustion tube and drying oven.

The entrance to the quartz combustion tube $(A$, fig. 3$)$ was closed by a Pyrex cap ground to fit tightly without a lubricant. By warming with the hand it expanded sufficiently to be removed. The oxygen

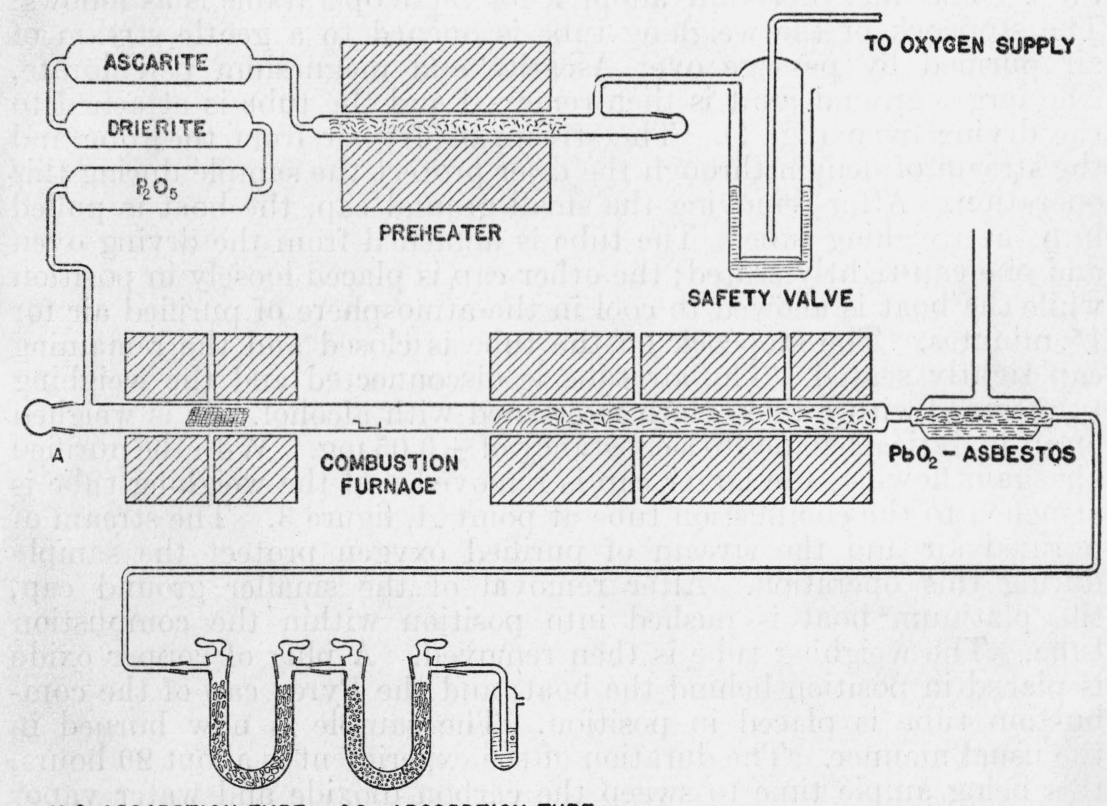

\section{$\mathrm{H}_{2} \mathrm{O}$ AESORPTION TUBE $\mathrm{CO}_{2}$ ABSORPTION TUBE}

FIGURE 3.-Apparatus for analysis of chars by combustion in oxygen. $A$, ground joint to fit weighing tube.

and the products of the combustion pass through the exit end via a quartz-to-Pyrex seal and then through a bed of lead peroxide [6] on asbestos which is maintained at $175^{\circ} \mathrm{C}$ by a Pyrex jacket containing refluxing $p$-cymene, as shown. The function of the lead peroxide is to remove the oxides of nitrogen and of sulfur which would otherwise 
introduce error by virtue of their retention by the Ascarite in the tared absorption tube for carbon dioxide. The absorption tubes were connected to the apparatus by means of interchangeable ground-glass joints and were packed as follows: First, magnesium perchlorate to retain the water vapor, and second, Ascarite followed by magnesium perchlorate to retain the carbon dioxide and water of neutralization. The ground joints of the absorption tubes were greased, and consequently, for each weighing the grease was removed with cotton swabs moistened with benzene. The tubes were then wiped with a cloth moistened with alcohol and weighed against a counterpoise of equivalent glass surface and volume.

\section{PROCEDURE}

A sample of 0.2 to $0.3 \mathrm{~g}$ of char is saturated with water vapor and allowed to dry in the stream of helium, as already described. One end of the weighing tube is closed by the larger ground joint which fits both the drying apparatus and the entrance joint on the quartz combustion tube. There are two stages to the transfer of the sample: (1) The loaded platinum boat is removed from the drying oven to be weighed, and (2) the boat is then transferred into the combustion tube. The final procedure adopted for these operations is as follows: The stopcock of the weighing tube is opened to a gentle stream of air purified by passage over Ascarite and magnesium perchlorate. The larger ground joint is then removed and the tube is attached to the drying oven (fig. 1). The stream of dry air from the tube and the stream of helium through the drier protect the sample during this operation. After removing the small ground cap, the boat is pulled into the weighing tube. The tube is detached from the drying oven and one cap tightly seated; the other cap is placed loosely in position while the boat is allowed to cool in the atmosphere of purified air for 15 minutes. The stopcock on the tube is closed and the remaining cap tightly seated. The air tube is disconnected and the weighing tube wiped with a linen cloth moistened with alcohol. It is weighed on an analy tical balance to a constancy of $\pm 0.05 \mathrm{mg}$. With the purified air again flowing, the larger cap is removed and the weighing tube is attached to the combustion tube at point $A$, figure 3 . The stream of purified air and the stream of purified oxygen protect the sample during this operation. After removal of the smaller ground cap, the platinum boat is pushed into position within the combustion tube. The weighing tube is then removed. A plug of copper oxide is placed in position behind the boat, and the Pyrex cap of the combustion tube is placed in position. The sample is now burned in the usual manner. The duration of the experiment is about 20 hours, this being ample time to sweep the carbon dioxide and water vapor through the apparatus.

\section{DETERMINATION OF CARBON DIOXIDE IN THE ASH FROM COMBUSTION AND IN THE ORIGINAL SUBSTANCE}

An alkaline ash remaining after the combustion of the sample, may retain a part of the evolved carbon dioxide in combination as carbonate. Hence, it is necessary to determine this carbon dioxide and to add it to the weight of carbon dioxide obtained by combustion. 
Also, the original char samples may contain some carbonate and possibly small quantities of firmly adsorbed carbon dioxide, which have already been included in the data from the combustion and from the ash analysis. Therefore, the ash from each combustion analysis and representative samples of the original char, prepared and dried in exactly the same manner as the samples for combustion, are analyzed for carbon dioxide by the following procedure.

The usual method [7] was used to determine the carbonate and the adsorbed carbon dioxide, namely, the evolution of carbon dioxide by the action of boiling dilute hydrochloric acid on the sample. In the case of this determination for the original substance, special precautions must be observed to prevent other evolved gases of acidic reaction, as well as water vapor, from reaching the absorption tube. The apparatus is illustrated by the diagram in figure 4 .

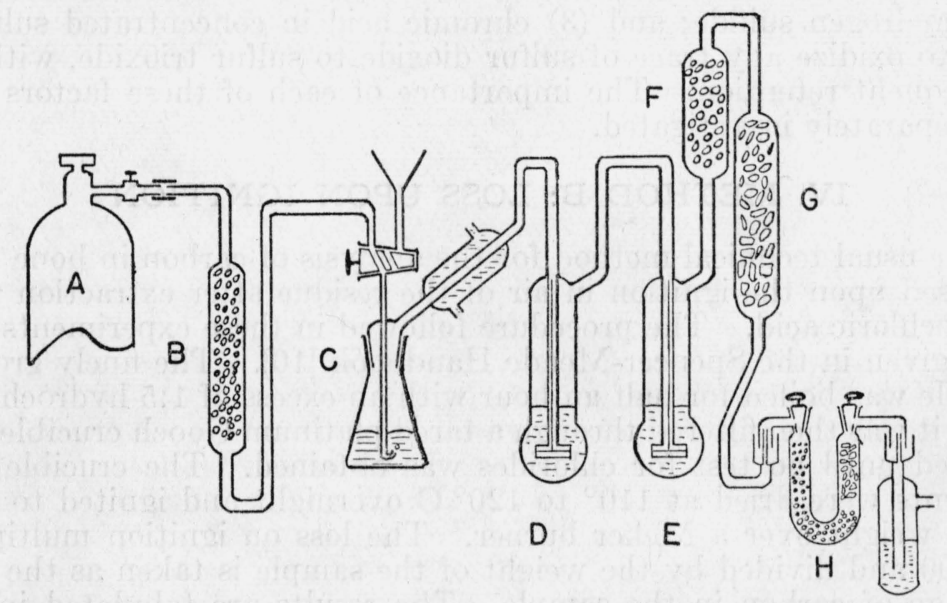

Figure 4.-Apparatus for determination of carbon dioxide in adsorbent materials.

$A$, nitrogen tank; $B$, soda-lime tube; $C$, Erlenmeyer flask with interchangeable ground joint; $D$, bubbler tube containing silver sulfate in sulfuric acid; $E$, bubbler tube containing chromic oxide in sulfuric acid; $F$, anhydrous copper sulfate on pumice stone; $G$, magnesium perchlorate drier; $H$, Ascarite and magnesium perchlorate for absorption of carbon dioxide.

The sample contained in the platinum boat was quickly transferred into a dry Erlenmeyer flask, which was immediately attached to the apparatus at the interchangeable joint, $C$. The apparatus was swept out for 5 minutes; then the sample was covered with distilled water, the acid added slowly, and the contents gradually brought to boiling: temperature. The gas stream passed through the water-jacketed condenser and then bubbled through a solution of silver sulfate in 1:1 sulfuric acid, thence through chromic acid in sulfuric acid, anhydrous copper sulfate on pumice stone, and magnesium perchlorate, as shown in figure 4. The carbon dioxide was retained in a tared tube filled with Ascarite followed by magnesium perchlorate. The bubbler tubes were of the special design described by Branham and Sperling [8]. The apparatus was swept out with nitrogen for 30 minutes, the absorption tube was detached, wiped, and weighed to a constancy of $\pm 0.05 \mathrm{mg}$ against a counterpoise of equivalent glass surface and volume. 
It was found that the use of air as the sweeping gas led to high blanks with the sample present in runs in which boiling water was used in place of the hydrochloric acid; the use of oxygen as the sweeping gas led to still higher blanks. However, water-pumped tank nitrogen eliminated this effect. It is not known just how extensive this slow oxidation of the sample might be in the presence of the hydrochloric acid when the sweeping gas contains oxygen. Experiments of Rideal and Wright [9] would indicate a poisoning effect of the hydrochloric acid operating to arrest auto-oxidation.

Considerable difficulty was at first experienced in obtaining concordant data by the above procedure. The three changes which furnished reproducible data were: (1) Substitution of nitrogen for air as the medium to sweep the evolved gases through the train; (2) both silver sulfate in concentrated sulfuric acid and anhydrous copper sulfate on pumice, in that order, to remove traces of hydrochloric acid and hydrogen sulfide; and (3) chromic acid in concentrated sulfuric acid to oxidize any trace of sulfur dioxide to sulfur trioxide, with its subsequent retention. The importance of each of these factors was not separately investigated.

\section{METHOD B: LOSS UPON IGNITION}

The usual technical method for the analysis of carbon in bone char is based upon the ignition in air of the residue after extraction with hydrochloric acid. The procedure followed in these experiments was that given in the Spencer-Meade Handbook [10]. The finely ground sample was boiled for half an hour with an excess of 1:5 hydrochloric acid; it was then filtered through a tared platinum Gooch crucible and washed until no test for chlorides was obtained. The crucible and contents were dried at $110^{\circ}$ to $120^{\circ} \mathrm{C}$ overnight and ignited to constant weight over a Meker burner. The loss on ignition multiplied by 100 and divided by the weight of the sample is taken as the percentage of carbon in the sample. The results are tabulated in the next section.

\section{DISCUSSION OF RESULTS}

The results of the analyses of several different chars of diverse physical and chemical characteristics are given in table 2 .

Extensive data on the carbon and hydrogen content of bone chars and other carbonaceous adsorbents are of interest primarily with reference to studies of the properties of the various chars. It was therefore deemed advisable to present here only certain representative data which indicate the compatibility of the two methods of analysis and the precision obtained.

Column 1 gives the identification numbers of the samples; column 2 , the type of char or carbon; columns 3 to 10 , inclusive, data from the analyses, expressed as percentages by weight of the original sample, column 3 giving the hydrogen content; the data in the remaining columns are as follows: 4 , carbon from combustion analysis; 5 , carbon in the carbonate of the ash; 6 , carbon in the carbonate and adsorbed carbon dioxide of the original sample; 7 , combustible carbon, i. e., 4 plus 5 minus average of $6 ; 8$ and 9 , the averaged corrected carbon values and the averaged hydrogen values, respectively; 10, the sum 
of combustible carbon and hydrogen, i. e., 8 plus 9 ; 11, the percentages of carbon as determined by the loss of weight upon the ignition of the acid-extracted residue; 12 , the percentage deviation of the lossupon-ignition method from the sum of the corrected carbon and hydrogen contents. These last columns are calculated from the mean of the two or more determinations for each sample.

TABLE 2.-Analysis of carbonaceous adsorbents

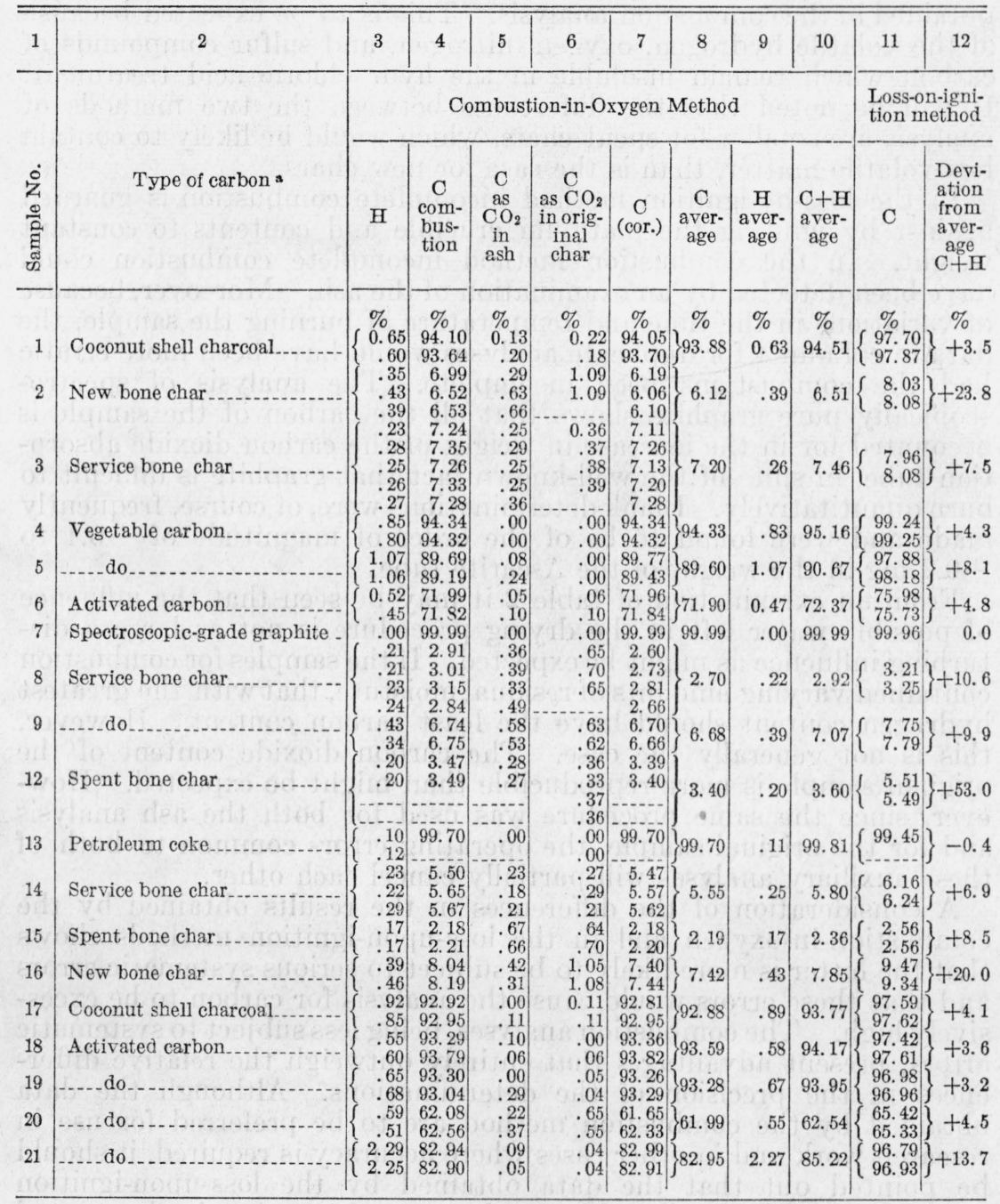

$0^{2}$ All 21 samples are products with different origins.

The net result given in column 7 is the quantity of combustible carbon. This would include the carbon content of any hydrocarbons as well as the elemental carbon. In the present research no attempt has been made to discriminate between the content of elemental carbon and of carbon in oxidizable states of combination, except insofar 
as the hydrogen content may serve as a rough indication of the possible content of hydrocarbons.

It is evident from the data in table 2 that the precision of the combustion data is not as good as that of the data obtained from the loss upon ignition. This situation may arise, in part, from possible errors in the auxiliary analyses for carbonates, which are necessary in the former method. The loss upon ignition would indicate a larger quantity of carbon than that determined from the weight of carbon dioxide obtained in the combustion analysis. This is to be expected because of the volatile hydrogen, oxygen, nitrogen, and sulfur compounds of carbon which remain insoluble in the hydrochloric acid treatment. It will be noted that the differences between the two methods of analysis are smaller for spent chars, which would be likely to contain less volatile matter, than is the case for new chars.

In the loss-by-ignition method incomplete combustion is guarded against by igniting the platinum crucible and contents to constant weight. In the combustion method incomplete combustion could have been detected by an examination of the ash. Moreover, because of variations in the time and temperature of burning the sample, the data in column 4 for duplicate analyses would have been more erratic had the combustions been incomplete. The analysis of spectroscopically pure graphite shows that all the carbon of the sample is accounted for in the increase in weight of the carbon dioxide absorption tube, in spite of the well-known fact that graphite is difficult to burn quantitatively. Blank determinations were, of course, frequently made and were found to be of the order of magnitude of -0.1 to $+0.3 \mathrm{mg}$ in the weight of the Ascarite tube.

From an examination of table 2 it may be seen that the influence of possible water left in the drying procedure is not as large a disturbing influence as might be expected. If the samples for combustion contained varying amounts of residual moisture, that with the greatest hydrogen content should have the least carbon content. However, this is not generally the case. The carbon dioxide content of the original sample is more reproducible than might be expected. However, since the same procedure was used for both the ash analysis and for the original sample, the operating errors common to both of these auxiliary analyses will partially cancel each other.

A consideration of the differences in the results obtained by the combustion-in-oxygen and in the loss-upon-ignition methods shows that the latter is more likely to be subject to serious systematic errors and that these errors would cause the analysis for carbon to be excessively high. The combustion analyses, being less subject to systematic errors, present advantages that entirely outweigh the relative differences in the precision of the determinations. Although the data obtained by the combustion method are to be preferred for use in research work and in other cases where accuracy is required, it should be pointed out that the data obtained by the loss-upon-ignition method are undoubtedly adequate for control purposes in the general routine of a sugar refinery laboratory. This is especially true if comparisons are to be made among service chars only or among new chars only. 
In the course of this work valued assistance has been received from C. J. Rodden, of the Chemistry Division, to whom we express our appreciation. We also express our appreciation to Eloise A. Swick, who was associated with us in this work.

\section{REFERENCES}

[1] M. E. Barker, Ind. Eng. Chem. 22, 926 (1930).

[2] F. M. Stanton, A. C. Fieldner, and W. A. Selvig, Tech. Pap. 8, Bureau of Mines, (1939)

[3] A. S. Coolidge, The adsorption of vapors by charcoal, J. Am. Chem. Soc. 46, 596 (1924).

[4] H. J. Wing, BS J. Research 10, 488 (1933).

[5] E. W. Washburn, J. H. Bruun, and M. M. Hicks, BS J. Research 2, 487 (1929).

[6] F. Pregl and H. Roth, Quantitative Organic Microanalyses, tr. by E. B. Daw, 3d English ed. (P. Blakiston's Son \& Co., Inc., Philadelphia, Pa., 1937).

[7] W. F. Hildebrand and G. E. F. Lundell, Applied Inorganic Analysis, page 623 (John Wiley \& Sons, Inc., New York, N. Y., 1929).

[8] J. R. Branham and E. O. Sperling, J. Research NBS R2, 701 (1939) RP1214.

[9] E. K. Rideal and W. M. Wright, J. Chem. Soc. 127, 1347 (1925).

[10] G. L. Spencer, A Handbook for Cane-Sugar Manufacturers and Their Chemists, page 184,7 th ed., revised by G. P. Meade (John Wiley \& Sons, Inc., New York, N. Y., 1929).

[11] J. A. Scherrer and G. E. F. Lundell, BS J. Research 5, 891 (1930) RP237.

WASHington, February 24, 1942. 


\section{MATHEMATICAL TABLES}

Attention is invited to a series of publications which is being prepared by the Project for the Computation of Mathematical Tables conducted by the Federal Works Agency, Work Projects Administration for the City of New York under the sponsorship of the National Bureau of Standards.

To date, eleven tables have been made available through the National Bureau of Standards. These are listed below:

MT1. Table or the First Ten Powers of the Integrrs From 1 to 1000:

(1938) VIII +80 pages; heavy paper cover. 50 cents.

MT2. Tables of the Exponential Function $e^{x}$ :

The ranges and intervals of the argument and the number of decimal places in the entries are given below:

$\begin{array}{ccc}\text { Range of } x & \text { Interval of } x & \text { Decimals given } \\ -2.5000 \text { to } 1.0000 & 0.0001 & 18 \\ 1.0000 \text { to } 2.5000 & .0001 & 15 \\ 2.500 \text { to } 5.000 & .001 & 15 \\ 5.00 \text { to } 10.0 & .01 & 12\end{array}$

(1939) XV +535 pages; bound in buckram, $\$ 2.00$.

MT3. Tables of Circular and Hyperbolic Sines and Cosines for Radian Arguments:

Contains 9 decimal place values of $\sin x, \cos x, \sinh x$ and $\cosh x$ for $x$ (in radians) ranging from 0 to 2 at intervals of 0.0001 .

(1939) XVII+ 405 pages; bound in buckram, $\$ 2.00$.

MT4. Tables of Sines and Cosines for Radian Arguments:

Contains 8 decimal place values of sines and cosines for radian arguments ranging from 0 to 25 at intervals of 0.001 .

(1940) XXIX + 275 pages; bound in buckram, $\$ 2.00$.

MT5. Tables of Sine, Cosine, and Exponential Intrgrals, volume I:

Values of these functions to 9 places of decimals from 0 to 2 at intervals of 0.0001 .

(1940) XXVI+444 pages; bound in buckram, $\$ 2.00$.

MT6. Tables of Sine, Cosine, and Exponential Intbgrals, Volume II:

Values of these functions to 9,10 , or 11 significant figures from 0 to 10 at intervals of 0.001 . with auxiliary tables.

(1940) XXXVII + 225 pages; bound in buckram, $\$ 2.00$.

MT7. Table of Natural Logarithms, Volumb I:

Logarithms of the integers from 1 to 50,000 to 16 places of decimals.

(1941) XVIII+ 501 pages; bound in buckram, $\$ 2.00$.

MT8. Tables of Probability Functions, Volume I:

Values of these functions to 15 places of decimals from 0 to 1 at intervals of 0.0001 and 1 to 5.6 at intervals of 0.001 .

(1941) XXVIII + 302 pages; bound in buckram, $\$ 2.00$.

MT9. TAble of Natural Logarithm, Volume II:

Logarithms of the integers from 50,000 to 100,000 to 16 places of decimals.

(1941) XVIII+501 pages; bound in buckram, $\$ 2.00$. 
MT10. Table of Natural Logarthms, Volume III:

Logarithms of the decimal numbers from 0.0001 to 5.0000 , to 16 places of decimals. (1941) XVIII + 501 pages; bound in buckram, $\$ 2.00$.

MT11. Tables of the Moments of Inertia and Section Moduli of Ordinary Angles, Channels, and Bulb Angles with Certain Platb Combinations.

(1941) XIII +197 pages; bound in green cloth. \$1.25.

Payment is required in advance. Make remittance payable to the "National Bureau of Standards", and send with order, using the blank form facing page 3 of the cover.

The prices are for delivery in the United States and its possessions and in countries extending the franking privilege. To other countries the price of MT1 is 65 cents; that of MT2, MT3, MT4, MT5, MT6, MT7, MT8, MT9, and MT10 is $\$ 2.50$ each; and that of MT11 is $\$ 1.50$; remittance to be made payable in United States currency.

Copies of these publications have been sent to various Government depositories throughout the country, such as public libraries in large cities, and colleges and universities, where they may be consulted.

A mailing list is maintained for those who desire to receive announcements regarding new tables as they become available. A list of the tables it is planned to publish will be sent on request. 